\section{Pharmacovigilance and Beyond}

\section{Adrian Preda*}

Health Sciences Professor, University of California Irvine, School of Medicine, USA

We are glad to report that the inception of the Journal of Pharmacovigilance has been greeted with enthusiasm, as reflected in a constant flux of submissions. We believe that open-access if the way of the future when it comes to scientific reports in general and Pharmacovigilance reports in particular.

In our opinion, medication safety is a public health issue and as such what better venue to address it than an open-access publication?

Of course our impact greatly depends on you, our readership. Please keep your submissions coming. While we cannot guarantee publication, as rigorous peer review always results in a fair number of rejections, we promise that no paper will be rejected without explanation. It is part of our mission to have our peer reviewers and editors help you improve the quality of your report independent of your decision to resubmit here or elsewhere.

In this issue we continue to address the concept of an expanded definition for Pharmacovigilance [1].

Drug induced hypersensitivity is an important clinical issue. Any patient can be sensitive to any drug and other than an informative discussion about the risk for a possible allergic reaction the assessment of hypersensitivity is usually completed by a "wait and see approach". Our poor ability to test prospectively for hypersensitivity results in not only in significant treatment delays but increases in costs related to both a decrease in efficacy (as an ideal efficacious drug would work the first time around, i.e. each drug failure amounts to a loss of efficacy) and in increase in toxicity. Teixeira and colleagues offer an elegant solution to this conundrum reporting that the assessment of CD69 and CD25 activation markers appears to be an useful prospective marker of drug allergy diagnosis in immediate and in delayed hypersensitivity.

While in most countries pharmaceutical products are closely monitored - including from a safety perspective -many other commercial products, containing active pharmaceutical ingredients, are offered to the public in a loosely regulated way. Such products are variously classified as supplements, vitamins, tonics or traditional medicines. In this context we appreciate Ozoua and Uwaya proposal of a systematic safety assessment for commercial herbal products starting, as it should, with animal safety data before moving into human testing.

Jankowski et al. report on the thiopurine S-methyltransferase phenotype and genotype implications for azathioprine treatment in children with inflammatory bowel disease making a case for the importance of a "hair splitting" strategy when it comes to the assessment of medications toxicity. The importance of the genomic signal for Pharmacovigilance studies cannot be underestimated at a time when high throughput omics technologies are starting to leave the high end academic laboratories for the clinic [2].

In her mini-review Parkill discusses the understudied issue of mucositis and stomatitis associated with conventional and targeted anticancer therapy. Parkill's report brings a new perspective to Pharmacovigilance reports, which traditional focus on "hard" adverse effects tends to skip over quality of life or "soft" treatment emergent issues. From the broader perspective of what it takes to cure cancer oncologists might see mucositis as a trivial adverse effect, a nuisance rather than a true concern. Reports such as Parkill's make a strong case that the importance of any adverse effect should ultimately by rated considering its impact on the patient's quality of life.

With this new selection of pharmacovigilance papers our journal is marching on.

\section{References}

1. A Preda (2013) Pharmacovigilance in the New Millennium: Challenges, Opportunities and New Directions. J Pharmacovigilance 1: e106.

2. Blankenburg M, Haberland L, Elvers HD, Tannert C, Jandrig B (2009) HighThroughput Omics Technologies: Potential Tools for the Investigation of Influences of EMF on Biological Systems. Curr Genomics 10: 86-92.
${ }^{*}$ Corresponding author: Adrian Preda, Health Sciences Professor, University of California Irvine, School of Medicine, USA, Tel: 714 456-8688; Fax: 714 456-5927; E-mail: apreda@uci.edu

Received September 30, 2013; Accepted October 03, 2013; Published October 11, 2013

Citation: Preda A (2013) Pharmacovigilance and Beyond. J Pharmacovigilance 1: e114. doi:10.4172/2329-6887.1000e114

Copyright: (c) 2013 Preda A. This is an open-access article distributed under the terms of the Creative Commons Attribution License, which permits unrestricted use, distribution, and reproduction in any medium, provided the original author and source are credited. 